\title{
Fixed Point Theorems and Error Bounds in Partial Metric Spaces
}

\author{
S. A. M. Mohsenalhosseini \\ Faculty of Mathematics, Vali-e-Asr University, Rafsanjan, Iran \\ Correspondence should be addressed to S. A. M. Mohsenalhosseini; mohsenialhosseini@gmail.com
}

Received 4 November 2013; Accepted 11 December 2013

Academic Editors: R. El-Khazali and Q.-W. Wang

Copyright (C) 2013 S. A. M. Mohsenalhosseini. This is an open access article distributed under the Creative Commons Attribution License, which permits unrestricted use, distribution, and reproduction in any medium, provided the original work is properly cited.

This paper is introduced as a survey of result on some generalization of Banach's fixed point and their approximations to the fixed point and error bounds, and it contains some new fixed point theorems and applications on dualistic partial metric spaces.

\section{Introduction}

The partial metric spaces were introduced in [1] as a part of the study of denotational semantics of dataflow networks. He established the precise relationship between partial metric spaces and the weightable quasi-metric spaces, and proved a partial metric generalization of Banach contraction mapping theorem.

A partial metric [1] on a set $X$ is a function $p: X \times X \rightarrow$ $[0, \infty)$ such that for all $x, y, z \in X$

(1) $x=y \Leftrightarrow p(x, x)=p(x, y)=p(y, y)$;

(2) $p(x, x) \leq p(x, y)$;

(3) $p(x, y)=p(y, x)$;

(4) $p(x, z) \leq p(x, y)+p(y, z)-p(y, y)$.

A partial metric space is a pair $(X, p)$, where $p$ is a partial metric on $X$. If $p$ is a partial metric on $X$, then the function $p^{s}: X \times X \rightarrow[0, \infty)$ given by $p^{s}(x, y)=2 p(x, y)-p(x, x)-$ $p(y, y)$ is a (usual) metric on $X$.

Each partial metric $p$ on $X$ induces a $T_{0}$ topology $\tau_{p}$ on $X$ which has as a basis the family of open $p$-balls $\left\{B_{p}(x, \epsilon): x \epsilon\right.$ $X, \epsilon>0\}$, where $B_{p}(x, \epsilon)=\{y \in X: p(x, y)<p(x, x)+\epsilon\}$ for all $x \in X$ and $\epsilon>0$. Similarly, closed $p$-ball is defined as $B_{p}(x, \epsilon)=\{y \in X: p(x, y) \leq p(x, x)+\epsilon\}$.

A sequence $\left\{x_{n}\right\}_{n \in N}$ in a partial metric space $(X, p)$ is called a Cauchy sequence if there exists (and is finite) $\lim _{n, m} p\left(x_{n}, x_{m}\right)[1]$.
Note that $\left\{x_{n}\right\}_{n \in N}$ is a Cauchy sequence in $(X, p)$ if and only if it is a Cauchy sequence in the metric space $\left(X, p^{s}\right)[1]$.

A partial metric space $(X, p)$ is said to be complete if every Cauchy sequence $\left\{x_{n}\right\}_{n \in N}$ in $X$ converges, with respect to $\tau_{p}$ to a point $x \in X$ such that $p(x, x)=\lim _{n, m} p\left(x_{n}, x_{m}\right)$ [1].

A mapping $T: X \rightarrow X$ is said to be continuous at $x_{0} \in$ $X$, if for $\epsilon>0$, there exists $\delta>0$ such that $T\left(B_{p}\left(x_{0}, \delta\right)\right) \subset$ $B_{p}\left(T\left(x_{0}\right), \epsilon\right)[2]$.

Definition 1 (see [1]). An open ball for a partial metric $p$ : $X \times X \rightarrow[0, \infty)$ is a set of the form $B_{\epsilon}^{p}(x):=\{y \in X$ : $p(x, y)<\epsilon\}$ for each $\epsilon>0$ and $x \in X$.

In [3], O'Neill proposed one significant change to Matthews definition of the partial metrics, and that was to extend their range from $R^{+}$to $R$. In the following, partial metrics in the O'Neill sense will be called dualistic partial metrics and a pair $(X, p)$ such that $X$ is a nonempty set and $p$ is a dualistic partial metric on $X$ will be called a dualistic partial metric space.

A dualistic partial metric on a set $X$ is a partial metric $p: X \times X \rightarrow R$. A dualistic partial metric space is a pair $(X, p)$, where $p$ is a dualistic partial metric on $X$.

A quasi-metric on a set $X$ is a nonnegative real-valued function $d$ on $X \times X$ such that for all $x, y, z \in X$

(i) $d(x, y)=d(y, x)=0 \Leftrightarrow x=y$,

(ii) $d(x, y) \leq d(x, z)+d(z, y)$. 
Lemma 2 (see $[1])$. If $(X, p)$ is a dualistic partial metric space, then the function $d_{p}: X \times X \rightarrow R^{+}$defined by $d_{p}(x, y)=$ $p(x, y)-p(x, x)$, is a quasi-metric on $X$ such that $\tau(p)=\tau\left(d_{p}\right)$.

Lemma 3 (see [1]). A dualistic partial metric space $(X, p)$ is complete if and only if the metric space $\left(X,\left(d_{p}\right)^{s}\right)$ is complete. Furthermore $\lim _{n \rightarrow \infty}\left(d_{p}\right)^{s}\left(a, x_{n}\right)=0$ if and only if $p(a, a)=$ $\lim _{n \rightarrow \infty} p\left(a, x_{n}\right)=\lim _{n, m \rightarrow \infty} p\left(x_{n}, x_{m}\right)$.

Before stating our main results, we establish some (essentially known) correspondences between dualistic partial metrics and quasi-metric spaces. Also refer to definition of $\epsilon$ Fixed point and the existence of $\epsilon$-Fixed point for $\epsilon>0$. Our basic references for quasi-metric spaces are $[4,5]$ and for $\epsilon$ Fixed point is [6].

If $d$ is a quasi-metric on $X$, then the function $d^{s}$ defined on $X \times X$ by $d^{s}(x, y)=\max \{d(x, y), d(y, x)\}$, is a metric on $X$.

Definition 4 (see [6]). Let $(X, p)$ be a dualistic partial metric space and $T: X \rightarrow X$ be a map. Then $x_{0} \in X$ is $\epsilon$-fixed point for $T$ if

$$
d_{p}\left(T x_{0}, x_{0}\right) \leq \epsilon
$$

We say $T$ has the $\epsilon$-fixed point property if for every $\epsilon>0$, $A F(T) \neq \emptyset$ where

$$
A F(T)=\left\{x_{0} \in X: d\left(T x_{0}, x_{0}\right) \leq \epsilon\right\} .
$$

Theorem 5 (see [6]). Let $(X, p)$ be a dualistic partial metric space and $T: X \rightarrow X$ be a map, $x_{0} \in X$ and $\epsilon>0$. If $d_{p}\left(T^{n}\left(x_{0}\right), T^{n+k}\left(x_{0}\right)\right) \rightarrow 0$ as $n \rightarrow \infty$ for some $k>0$, then $T^{k}$ has an $\epsilon$-fixed point.

Definition 6 (see [7]). Let $T: A \cup B \rightarrow A \cup B$, be continues map such that $T(A) \subseteq B, T(B) \subseteq A$ and $\epsilon>0$. We define diameter $P_{T}^{a}(A, B)$ by

$$
\operatorname{diam}\left(P_{T}^{a}(A, B)\right)=\sup \left\{d(x, y): x, y \in P_{T}^{a}(A, B)\right\} .
$$

Theorem 7 (see [1]). The partial metric contraction mapping theorem. Let $(X, p)$ be a complete partial metric space and $T$ : $X \rightarrow X$ be a map such that for all $x, y \in X$

$$
p(T x, T y) \leq L p(x, y): \quad 0 \leq L<1,
$$

then $T$ has a unique fixed point $u$, and $T^{n}(x) \rightarrow u$ as $n \rightarrow \infty$ for each $x \in X$.

\section{Some Result Fixed Point on Partial Metric}

In this section, we give some result on fixed point and $\epsilon$-fixed point in dualistic partial metric space and its diameter.

Definition 8. An open ball for a dualistic partial metric $p$ : $X \times X \rightarrow R$ is a set of the form $B_{\epsilon}^{p}(x):=\{y \in X: p(x, y)<$ $\epsilon\}$ for each $\epsilon>0$ and $x \in X$.

Definition 9. Let $T$ be a mapping of a complete dualistic partial metric $X$ into itself $[T: X \rightarrow X]$, then $T$ is called a partial metric contraction mapping if there exists a constant $L, 0 \leq L<1$, such that $p(T x, T y) \leq L p(x, y)$ for all $x, y \in X$.

Theorem 10. Every contraction mapping $T$ defined on a complete dualistic partial metric $X$ into itself has a unique fixed point $u \in X$. Moreover, if $x_{0}$ is any point in $X$ and the sequence $x_{n}$ is defined by

$$
x_{1}=T\left(x_{0}\right), \quad x_{2}=T\left(x_{1}\right), \ldots, x_{n}=T\left(x_{n-1}\right),
$$

then $\lim _{n \rightarrow \infty} x_{n}=u$ and

$$
p\left(x_{n}, u\right) \leq \frac{L^{n}}{1-L} p\left(x_{1}, x_{0}\right): \quad 0 \leq L<1 .
$$

Proof. Existence of a fixed point. Let $x_{0}$ be an arbitrary point in $X$, and we defined by $x_{1}=T\left(x_{0}\right), x_{2}=T\left(x_{1}\right), \ldots, x_{n}=$ $T\left(x_{n-1}\right)$. Then,

$$
\begin{aligned}
& x_{2}=T\left(x_{1}\right)=T\left(T\left(x_{0}\right)\right)=T^{2}\left(x_{0}\right) \\
& x_{3}=T\left(x_{2}\right)=T\left(T\left(x_{1}\right)\right)=T\left(T\left(T\left(x_{0}\right)\right)\right)=T^{3}\left(x_{0}\right)
\end{aligned}
$$

$x_{n}=T^{n}\left(x_{0}\right)$.

If $m>n$, say $m=n+\alpha, \alpha=1,2,3, \ldots$.

Then

$$
\begin{aligned}
p\left(x_{n}+\alpha, x_{n}\right) & =p\left(T^{n+\alpha}\left(x_{0}\right), T^{n}\left(x_{0}\right)\right) \\
& =p\left(T\left(T^{n+\alpha-1}\left(x_{0}\right)\right), T\left(T^{n-1}\left(x_{0}\right)\right)\right) \\
& \leq L p\left(T^{n+\alpha-1}\left(x_{0}\right), T^{n-1}\left(x_{0}\right)\right) .
\end{aligned}
$$

Continuing this process $n-1$ times, we have

$$
p\left(x_{n+\alpha}, x_{n}\right) \leq L^{n} p\left(T^{\alpha}\left(x_{0}\right), x_{0}\right)
$$

for $n=0,1,2, \ldots$, and all $\alpha \geq 1$.

However,

$$
\begin{aligned}
p\left(T^{\alpha}\left(x_{0}\right), x_{0}\right) \leq & p\left(T^{\alpha}\left(x_{0}\right), T^{\alpha-1}\left(x_{0}\right)\right) \\
& +p\left(T^{\alpha-1}\left(x_{0}\right), T^{\alpha-2}\left(x_{0}\right)\right)+\cdots \\
& +p\left(T\left(x_{0}\right), x_{0}\right) .
\end{aligned}
$$

Therefore, we see that

$$
\begin{aligned}
& p\left(x_{n+\alpha}, x_{n}\right) \\
& \quad \leq L^{n}\left[L^{\alpha-1} p\left(x_{1}, x_{0}\right)+L^{\alpha-2} p\left(x_{1}, x_{0}\right)+\cdots+p\left(x_{1}, x_{0}\right)\right] \\
& \quad \leq L^{n} p\left(x_{1}, x_{0}\right)\left[1+L+L^{2}+\cdots+L^{\alpha-1}+L^{\alpha}\right] .
\end{aligned}
$$

Hence,

$$
p\left(x_{n+\alpha}, x_{n}\right) \leq L^{n} p\left(x_{1}, x_{0}\right) \frac{1}{1-L} \text {. }
$$


As $n, m=n+\alpha \rightarrow \infty$, from (12), we see that $p\left(x_{n+\alpha}, x_{n}\right) \rightarrow 0$; that is, $\left\{x_{n}\right\}$ is a Cauchy sequence in the metric space $X$. Hence, $\left\{x_{n}\right\}$ must be convergent, say $\lim _{n \rightarrow \infty} x_{n}=u$.

Since $T$ is continuous, we have

$$
T u=T\left(\lim _{n \rightarrow \infty} x_{n}\right)=\lim _{n \rightarrow \infty} T\left(x_{n}\right)=\lim _{n \rightarrow \infty} x_{n+1}
$$

or $T u=u$. Thus, $u$ is a fixed point of $T$.

Uniqueness of the fixed point $T$.

Let $v$ be another fixed point of $T$. Then $T v=v$. We also have $p(T(u), T(v)) \leq L p(u, v)$. But $p(T(u), T(v))=p(u, v)$ which implies that $p(u, v) \leq L p(u, v)$ where $0 \leq L<1$. This is possible only when $p(u, v)=0$, that is, $u=v$. This proves that the fixed point of $T$ is unique.

Corollary 11. Let $(X, p)$ be a complete dualistic partial metric space and $B_{r}^{p}\left(x_{0}\right):=\left\{y \in X: p\left(x_{0}, y\right)<r\right\}$. Let $T: B_{r}^{p} \rightarrow X$ be a partial metric contraction mapping If $p\left(T y_{0}, y_{0}\right)<(1-$ $L) r$, then $T$ has a fixed point.

Proof. Choose $r<\epsilon$ so that $p\left(T y_{0}, y_{0}\right) \leq(1-L) r<(1-L) \epsilon$. We show that $T$ maps the closed ball $K=\left\{y: p\left(y, y_{0}\right) \leq \epsilon\right\}$ into itself; for if $y \in K$, then

$$
\begin{aligned}
p\left(T(y), y_{0}\right) & \leq p\left(T(y), T\left(y_{0}\right)\right)+p\left(T\left(y_{0}\right), y_{0}\right) \\
& \leq L p\left(y, y_{0}\right)+(1-L) \epsilon \\
& \leq L \epsilon+\epsilon-L \epsilon=\epsilon .
\end{aligned}
$$

Since $K$ is complete and $T: K \rightarrow K$ satisfy in (5) thus by Theorem 10, $T$ has a fixed point

Theorem 12. If $X$ is a complete dualistic partial metric space, and $T: X \rightarrow X$ is such that $T^{r}$ is contraction for some integer $r>0$, then $T^{r}$ has an unique fixed point.

Proof. Since $T^{r}$, where $r$ is a positive integer, is a contraction mapping by Theorem 10 , there exists an unique fixed point $u$ of $T^{r}$, that is, $T^{r}(u)=u$. We want to show that $u$ ia a fixed point of $T$, that is, $T(u)=u$. Let $S=T^{r}$; therefore, $S(u)=u$. This implies that

$$
\begin{gathered}
S^{2}(u)=S(S(u))=S(u)=u, \\
S^{3}(u)=S^{2}(S(u))=S^{2}(u) h=u, \\
S^{n}(u)=S^{n-1}(S(u))=S^{n-1}(u)=\cdots=u,
\end{gathered}
$$

and so $T(u)=T\left(S^{n}(u)\right)=s^{n}(T(u))=s^{n} y$ say

$$
\begin{aligned}
p\left(S^{n}(y), u\right) & =p\left(S^{n}(y), S^{n}(u)\right) \\
& \leq L p\left(S^{n-1}(y), S^{n-1}(u)\right)
\end{aligned}
$$

$$
\begin{aligned}
& \leq L^{2} p\left(S^{n-2}(y), S^{n-2}(u)\right) \\
& \vdots \\
& \leq L^{n} p(y, u), \\
& \lim _{n \rightarrow \infty} p\left(S^{n}(y), u\right)=0,
\end{aligned}
$$

as $L^{n} \rightarrow 0$. Thus, $\lim _{n \rightarrow \infty} S^{n}(y)=u$ and we have $T(u)=$ $u$.

Theorem 13. Let $(X, p)$ is a complete dualistic partial metric space, and let $T: X \rightarrow X$ and $S: X \rightarrow X$ be two maps contraction. If for every $x \in X$,

$$
p(T x, S y) \leq \lambda
$$

$\lambda>0$, chosen suitably. Then for every $x \in X$,

$$
\begin{array}{r}
p\left(T^{m}(x), S^{m}(x)\right) \leq \lambda \frac{1-L^{m}}{1-L}: \quad 0 \leq L<1, \\
m=1,2, \ldots .
\end{array}
$$

Proof. The relation is true for $m=1$. We use the principle of induction in order to prove this relation. Let it be true for all $m \geq 1$. Then

$$
\begin{aligned}
p\left(T^{n+1}(x)-S^{n+1}(x)\right)= & p\left(T T^{m}(x), S S^{m}(x)\right) \\
\leq & p\left(T T^{m}(x), T S^{m}(x)\right) \\
& +p\left(T S^{m}(x), S S^{m}(x)\right) \\
\leq & L p\left(T^{m}(x), S^{m}(x)\right)+\lambda \\
\leq & L \lambda \frac{1-L^{m}}{1-L}+\lambda \\
= & \frac{L \lambda-L^{m+1} \lambda+\lambda-L \lambda}{1-L} \\
= & \lambda \frac{1-L^{m+1}}{1-L} .
\end{aligned}
$$

Thus, the relation is true for $m+1$.

Corollary 14. Let $(X, p)$ is a complete dualistic partial metric space and let $T: X \rightarrow X$ be a partial contractive map on $X$. Moreover, the iteration sequence $x_{1}=T\left(x_{0}\right), x_{2}=T\left(x_{1}\right)=$ $T^{2} x_{0}, \ldots, x_{n}=T^{n} x_{0}, \ldots$ with arbitrary $x_{0} \in X$ converges to the unique fixed point $u$ of $T$. Error estimate is the following estimate (prior estimate):

$$
p\left(x_{m}, u\right) \leq \frac{L^{m}}{1-L} p\left(x_{0}, x_{1}\right)
$$

and the posterior estimate

$$
p\left(x_{m}, u\right) \leq \frac{L}{1-L} p\left(x_{m-1}, x_{m}\right) .
$$


Proof. The First statement is obvious by

$$
\begin{aligned}
p\left(x_{m}, x_{n}\right) \leq & p\left(x_{m}, x_{m+1}\right)+p\left(x_{m+1}, x_{m+2}\right)+\cdots \\
& +p\left(x_{n-1}, x_{n}\right) \\
\leq & \left(L^{m}+L^{m+1}+\cdots+L^{n-1}\right) p\left(x_{0}, x_{1}\right) .
\end{aligned}
$$

Thus

$$
p\left(x_{m}, x_{n}\right) \leq \frac{L^{m}}{1-L} p\left(x_{0}, x_{1}\right) \quad(n>m) .
$$

Now, inequality (20) follows from (23) by letting $n \rightarrow \infty$. We have

$$
p\left(x_{m}, u\right) \leq \frac{L^{m}}{1-L} p\left(x_{0}, x_{1}\right) .
$$

Now, for inequality (21) taking $m=1$ and writing $y_{0}$ for $x_{0}$ and $y_{1}$ for $x_{1}$, we have from (23)

$$
p\left(y_{1}, u\right) \leq \frac{L}{1-L} p\left(y_{0}, y_{1}\right) .
$$

Setting $y_{0}=x_{m-1}$, we have $y_{1}=T y_{0}=x_{m}$ and obtain (21).

Corollary 15. Let $(X, p)$ be a complete dualistic partial metric space and let $T: X \rightarrow X$ be a contraction on a closed ball $B=B\left(x_{0}, r\right)=\left\{x: p\left(x, x_{0}\right) \leq r\right\}$. Moreover, assume that $p\left(x_{0}, T x_{0}\right)<(1-L) r$. Then, prior error estimate is the following estimate:

$$
p\left(x_{m}, u\right) \leq L^{m} r
$$

and the posterior estimate

$$
p\left(x_{m}, u\right) \leq L r .
$$

Proof. By Corollary 11 the iteration sequence as (5) is converges to the unique fixed point $u$ of $T$; hence, by Corollary 14 , we have

$$
p\left(x_{m}, u\right) \leq \frac{L^{m}}{1-L} p\left(x_{0}, x_{1}\right)
$$

since $x_{1}=T x_{0}$ and $p\left(x_{0}, T x_{0}\right)<(1-L) r$, we have

$$
\begin{aligned}
p\left(x_{m}, u\right) & \leq \frac{L^{m}}{1-L} p\left(x_{0}, x_{1}\right) \\
& =\frac{L^{m}}{1-L} p\left(x_{0}, T x_{0}\right) \\
& <\frac{L^{m}}{1-L} \cdot(1-L) r \\
& =L^{m} \cdot r .
\end{aligned}
$$

Therefore $p\left(x_{m}, u\right)<L^{m} r$. Also, by Corollary 14 we have

$$
p\left(x_{m}, u\right) \leq \frac{L}{1-L} p\left(x_{m-1}, x_{m}\right),
$$

since $x_{m-1}=T^{m-1} x_{0}$ and $p\left(x_{0}, T x_{0}\right)<(1-L) r$, we have

$$
\begin{aligned}
p\left(x_{m}, u\right) & \leq \frac{L}{1-L} p\left(x_{m-1}, x_{m}\right) \\
& =\frac{L}{1-L} p\left(T^{m-1} x_{0}, T^{m} x_{0}\right) \\
& =\frac{L}{1-L} p\left(T^{m-1} x_{0}, T\left(T^{m-1} x_{0}\right)\right) \\
& <\frac{L}{1-L}(1-L) r \\
& <L r .
\end{aligned}
$$

Therefore $p\left(x_{m}, u\right)<L r$.

\section{Applications of Banach Contraction Principle on Complete Dualistic Partial Metric Space}

In this section, we apply Theorem 10 to prove existence of the solutions a system of $n$ linear algebraic equations with $n$ unknowns, and we show that applied of Corollaries 14 and 15 in numerical analysis.

3.1. Application 3.1. Suppose we want to find the solution of a system of $n$ linear algebraic equations with $n$ unknowns, then

$$
\begin{aligned}
& a_{11} x_{1}+a_{12} x_{2}+\cdots+a_{1 n} x_{n}=b_{1} \\
& a_{21} x_{1}+a_{22} x_{2}+\cdots+a_{2 n} x_{n}=b_{2}
\end{aligned}
$$

$$
a_{n 1} x_{1}+a_{n 2} x_{2}+\cdots+a_{n n} x_{n}=b_{n} \text {. }
$$

This system can be written as

$$
\begin{gathered}
x_{1}=\left(1-a_{11}\right) x_{1}-a_{12} x_{2}-a_{13} x_{3}-\cdots-a_{1 n} x_{n}+b_{1} \\
x_{2}=-a_{21} x_{1}+\left(1-a_{22}\right) x_{2}-a_{23} x_{3}-\cdots-a_{2 n} x_{n}+b_{2} \\
x_{3}=-a_{31} x_{1}-a_{3} 2 x_{3}+\left(1-a_{33}\right) x_{3}-\cdots-a_{3 n} x_{n}+b_{3} \\
\vdots \\
x_{n}=-a_{n 1} x_{1}-a_{n 2} x_{2}-a_{n 3} x_{3}-\cdots+\left(1-a_{n n}\right) x_{n}+b_{n} .
\end{gathered}
$$

By assuming $\alpha_{i j}=-a_{i j}+\delta_{i j}$, where

$$
\delta_{i j}= \begin{cases}0 & i \neq j \\ 1 & i=j .\end{cases}
$$

Equation (33) can be written in the following equivalent form:

$$
x_{i}=\sum_{j=1}^{n} \alpha_{i j} x_{j}+b_{i}, \quad i=1,2,3, \ldots, n .
$$


If $x=\left(x_{1}, x_{2}, \ldots, x_{n}\right) \in R^{n}$ then (35) can be written in the form $T x=x$, where $T$ is defined by

$$
\begin{aligned}
T x & =y, \quad \text { where } y=\left(y_{1}, y_{2}, \ldots, y_{n}\right), \\
y_{i} & =\sum_{j=1}^{n} \alpha_{i j} x_{j}+b_{i}, \quad i=1,2,3, \ldots, n,
\end{aligned}
$$

$T: R^{n} \longrightarrow R^{n}, \quad\left(\alpha_{i j}\right)$ is a $n \times n$ matrix.

Finding solutions of the system described by (32) or (35) is thus equivalent to finding the fixed point of the operator equation, (36). In order to find a unique solution of $T$, that is, a unique solution of (32), we apply Theorem 10. In fact, we prove the following result. Equation (32) has a unique solution, if

$$
\sum_{j=1}^{n}\left|\alpha_{i j}\right|=\sum_{j=1}^{n}\left|-a_{i j}+\delta_{i j}\right| \leq L<1, \quad i=1, \ldots, n .
$$

For $x=\left(x_{1}, \ldots, x_{n}\right)$ and $x^{\prime}=\left(x_{1}^{\prime}, x_{2}^{\prime}, \ldots, x_{n}^{\prime}\right)$, we have

$$
p\left(T x, T x^{\prime}\right)=p\left(y, y^{\prime}\right)
$$

where

$$
\begin{gathered}
y=\left(y_{1}, y_{2}, \ldots, y_{n}\right) \in R^{n}, \\
y^{\prime}=\left(y_{1}^{\prime}, y_{2}^{\prime}, \ldots, y_{n}^{\prime}\right) \in R^{n}, \\
y_{i}=\sum_{j=1}^{n} \alpha_{i j} x_{j}+b_{i}, \\
y_{i}^{\prime}=\sum_{j=1}^{n} \alpha_{i j} x_{j}^{\prime}+b_{i}, \quad i=1,2, \ldots, n .
\end{gathered}
$$

If $y=\left(y_{1}, y_{2}, \ldots, y_{n}\right) \in R^{n}$, then $p(y, y)=\sup _{1 \leq i \leq n}\left|y_{i}\right|$. Therefore,

$$
\begin{aligned}
p\left(T x, T x^{\prime}\right) & =\sup _{1 \leq i \leq n}\left|y_{i}-y_{i}^{\prime}\right| \\
& =\sup _{1 \leq i \leq n}\left|\sum_{j=1}^{n} \alpha_{i j} x_{j}+b_{i}-\sum_{j=1}^{n} \alpha_{i j} x_{j}^{\prime}-b_{i}\right| \\
& =\sup _{1 \leq i \leq n}\left|\sum_{j=1}^{n} \alpha_{i j}\left(x_{j}-x_{j}^{\prime}\right)\right| \\
& \leq \sup _{1 \leq i \leq n} \sum_{j=1}^{n}\left|\alpha_{i j}\right|\left|x_{j}-x_{j}^{\prime}\right| \\
& \leq \sup _{1 \leq j \leq n}\left|x_{j}-x_{j}^{\prime}\right| \sup _{1 \leq i \leq n} \sum_{j=1}^{n}\left|\alpha_{i j}\right| \\
& \leq \sup _{1 \leq j \leq n}\left|x_{j}-x_{j}^{\prime}\right| .
\end{aligned}
$$

Since $p\left(x, x^{\prime}\right)=\sup _{1 \leq j \leq n}\left|x_{j}-x_{j}^{\prime}\right|$, we have $p\left(T x, T x^{\prime}\right) \leq$ $L p\left(x, x^{\prime}\right), 0 \leq L<1$, that is, $T$ is a contraction mapping of the complete dualistic partial metric space $R^{n}$ into itself. Hence, by Theorem 10, there exists a unique fixed point $u$ of $T$ in $R^{n}$, that is, $u$ is a unique solution of (32).

Theorem 16. If $f(t)$ is a nonlinear integral equation as the following:

$$
f(t)=\int_{0}^{t} e^{-v t} \cos (\alpha f(v)) d v, \quad 0 \leq t \leq 1 ; 0<\alpha<1,
$$

then it has a unique solution.

Proof. We apply Theorem 10 and we can prove that this equation has a unique continuous real-valued solution $f(t)$. Let $X=C[0,1]$ and the mapping $T: X \rightarrow X$, defined by $T(f)=f$ for $f \in X$, where $X$ is a complete dualistic partial metric space with sup $p(x, y)$, is a contraction mapping:

$$
\cos (\alpha a)-\cos (\alpha b)=\alpha(b-a) \sin \beta,
$$

where $\beta$ lies between $\alpha a$ and $\alpha b$. Therefore, $\mid \cos (\alpha a)-$ $\cos (\alpha b)|\leq \alpha| b-a \mid$. For functions $a(t)$ and $b(t)$; we get

$$
|\cos \alpha a(t)-\cos \alpha b(t)| \leq \sup _{0 \leq t \leq 1}|a(t)-b(t)|=p(a, b) .
$$

For $f=T f$ and $g=T g$, we have

$$
\begin{aligned}
|T f-T g| & =\left|\int_{0}^{t} e^{-v t}\right| \cos (\alpha f(v))-\cos (\alpha g(v))|d v| \\
& \leq \int_{0}^{t} e^{-v t}|\cos (\alpha f(v))-\cos (\alpha g(v))| d v \\
& \leq \alpha p(f, g) \int_{0}^{t} e^{-v t} d v \leq \alpha p(f, g) .
\end{aligned}
$$

Taking sup over $0 \leq t \leq 1$, we get

$$
\sup _{t}|T f(t)-T g(t)| \leq \alpha p(f, g)
$$

or

$$
p(T(f), T(g)) \leq \alpha p(f, g)
$$

Theorem 17. Let $x_{0}$ be an initial value and the iterative sequence $\left\{x_{n}\right\}$ as the following:

$$
x_{n}=g\left(x_{n-1}\right) \quad n=1,2, \ldots
$$

If $g$ is continuously differentiable on some interval $K=\left[x_{0}-\right.$ $\left.r, x_{0}+r\right]$ and satisfies $\left|g^{\prime}(x)\right| \leq L<1$ on $K$ as well as

$$
\left|g\left(x_{0}\right)-x_{0}\right|<(1-L) r
$$

then $x=g(x)$ has a unique solution $u$ on $K$, the iterative sequence $\left\{x_{m}\right\}$ converges to that solution, and one has the error estimates

$$
\left|x-x_{m}\right|<L^{m} r, \quad\left|x-x_{m}\right|<L r .
$$


Proof. Suppose that $p(x, g(x))=|x-g(x)|$ for $x \in K$. By the mean-value theorem and the given condition, $g(x)$ is a contraction mapping of the complete dualistic partial metric space $K$ into itself. Hence, by Corollary 11, there exists a unique fixed point $u$ of $g$ in $K$, that is, $u$ is a unique solution of $x=g(x)$. Also, the iteration sequence $\left\{x_{m}\right\}$ converges to $u$. Moreover, by $p(x, g(x))=|x-g(x)|$ and Corollary 15, it has the prior error estimate

$$
\left|x-x_{m}\right| \leq L^{m} r
$$

and the posterior estimate

$$
\left|x-x_{m}\right| \leq \operatorname{Lr}
$$

\section{References}

[1] S. G. Matthews, "Partial metric topology," in Proceedings of the 11th Summer Conference on General Topology and Applications, vol. 728, pp. 183-197, The New York Academy of Sciences, Augusts 1995.

[2] I. Altun and A. Erduran, "Fixed point theorems for monotone mappings on partial metric spaces," Fixed Point Theory and Applications, vol. 2011, Article ID 508730, 10 pages, 2011.

[3] S. J. O'Neill, "Partial metrics, valuations, and domain theory," Annals of the New York Academy of Sciences, vol. 806, pp. 304315, 1996.

[4] P. Fletcher and W. F. Lindgren, Quasi-Uniform Spaces, Marcel Dekker, New York, NY, USA, 1982.

[5] H. P. A. Kunzi, "Handbook of the history of general topology," in Non-Symmetric Distances and Their Associated Topologies: About the Origins of Basic Ideas in the Area of Asymmetric Topology, vol. 3, pp. 853-968, Kluwer Academic, 2001.

[6] S. A. M. Mohsenalhosseini, H. Mazaheri, M. A. Dehghan, and A. Zareh, "Fixed point for partial metric spaces," ISRN Applied Mathematics, vol. 2011, Article ID 657868, 6 pages, 2011.

[7] S. A. M. Mohsenalhosseini, H. Mazaheri, and M. A. Dehghan, "Approximate best proximity pairs in metric space," Abstract and Applied Analysis, vol. 2011, Article ID 596971, 9 pages, 2011. 


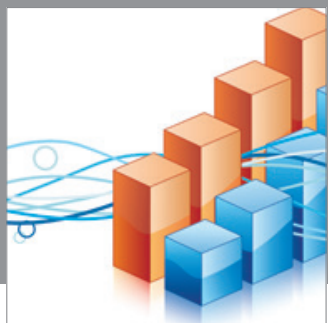

Advances in

Operations Research

mansans

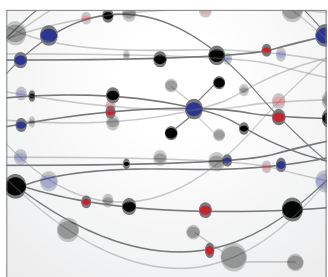

The Scientific World Journal
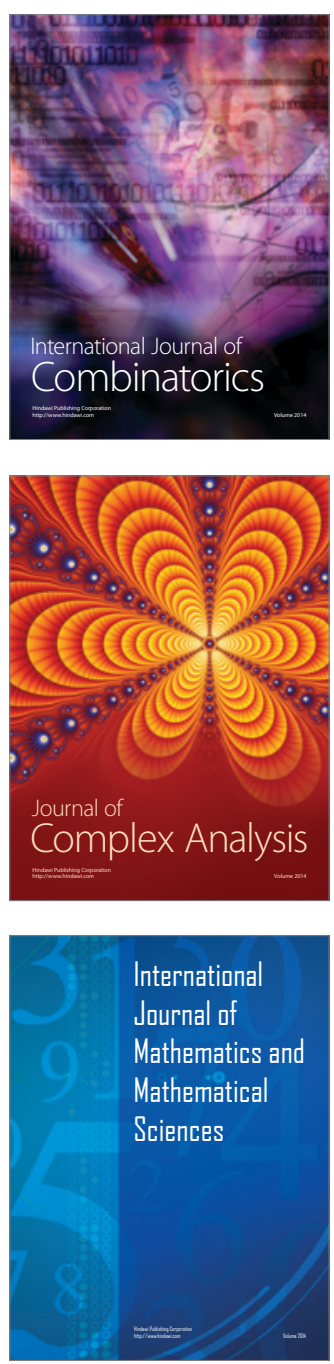
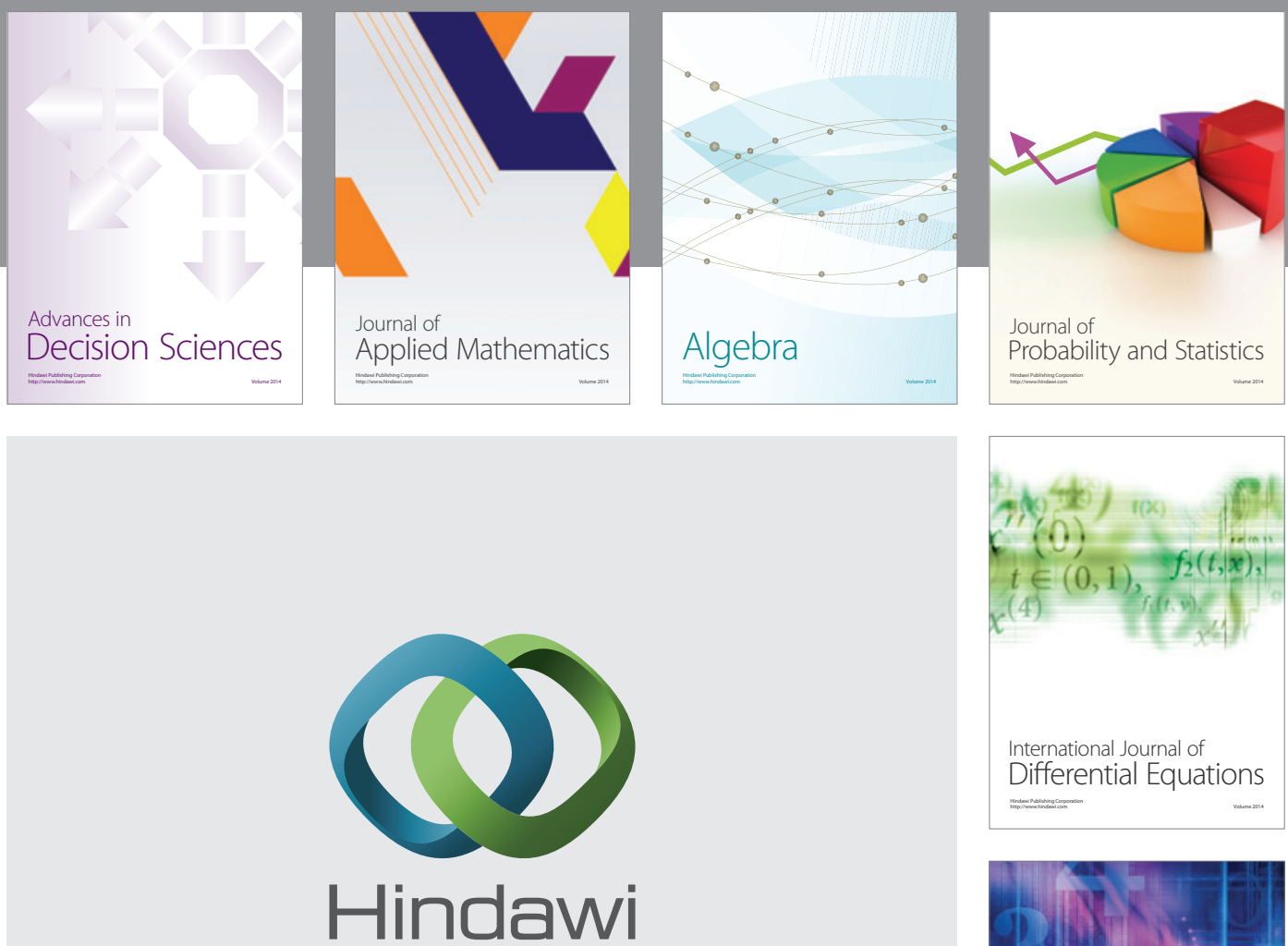

Submit your manuscripts at http://www.hindawi.com
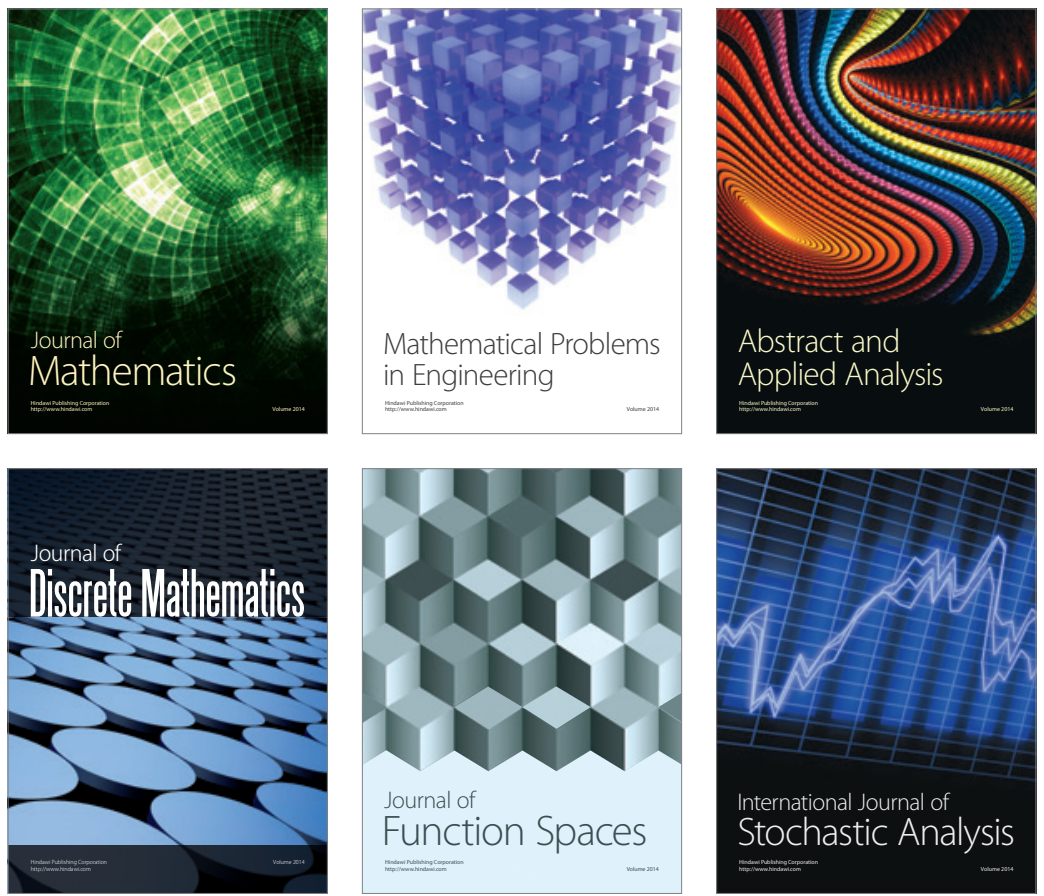

Journal of

Function Spaces

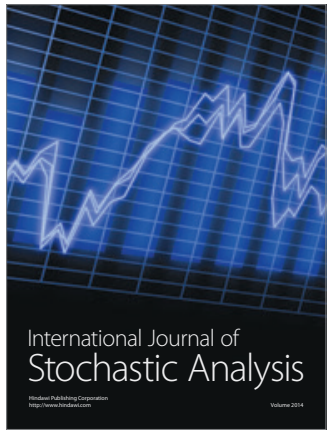

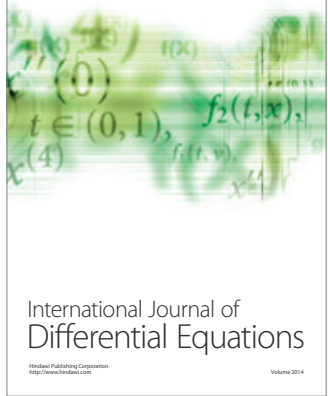
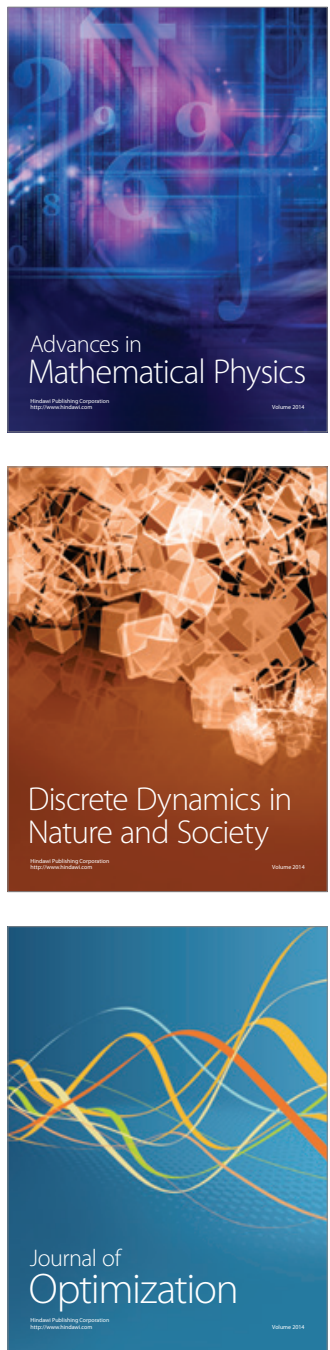